\title{
A Sequential Response Surface Method Using I nherted Uniform Design
}

\author{
Sun Shiping \\ School of aeronautical manufacturing engineering \\ Nanchang Hangkong University \\ Nanchang, China \\ sunsping@126.com
}

\author{
Hu Jiantang \\ School of aeronautical manufacturing engineering \\ Nanchang Hangkong University \\ Nanchang, China \\ 895220181@qq.com
}

\begin{abstract}
This study presents a Sequential quadratic Response Surface Method(SRSM) using inherited Uniform Design(UD), to reduce the computational burden in optimization process and improve the computational efficiency of engineering optimization. The program comprises a low accuracy global quadratic response surface modeling and a precision increasing sequential quadratic response surface modeling, which were consturcted by the 1.5 and 1 times saturated sampling points, respectively. The initial sub-region of the sequential response surface is determined by the global response surface, according to the optimum point in the sample set. A new move limit criterion based on the relative position of normalized optimum is proposed to achieve sub-region planning and zooming. Three test mathematic functions and a buckling optimization design problem are solved to show the numerical performance of the proposed method. The calculated data show that the inherited UD and the move limit criterion introduced into approximate modeling can effectively improve the efficiency and the conergence accuracy of the method presented. The results exhibit the progressive in the computation-inte nsive engineering application.
\end{abstract}

Keywords- response surface method; uniform design; move limit; buckling optimization; modeling method

\section{INTRODUCTION}

As today's engineering design have widely used the optimization method to improve the structure performances, many elaborate analyses and simulation are necessary for design process. It is a very time consuming process if design analysis and simulation is computationintensive. Especially an optimization process combined with direct search algorithm becomes more expensive, which needs a great many analyses. In order to raise an efficiency of design process by reducing the number of computation-intensive analyses, Response Surface Method (RSM) was commonly introduced to approximate complex system. RSM is one of the most popular methods to generate the explicit approximate expression instead of the implicit relationship between the inputs and outputs of complex system, based on the experimental design and the statistical principle. For the optimization design using RSM, the system responses are only conducted at the sampling points, and others system analyses use the approximate model instead of computation-intensive analyses, so the simulation time is saved. But RSM has two disadvantages[1]. First, as the increase of design variables, the number of sampling points would increase exponentially. It leads the optimization to the lower efficiency. Secondly, it's difficult to get a global optimum via a quadratic response surface model when the problem is highly non-linear, because the low order response surface model cannot estimate the non-linearity accurately. Current RSM related research is roughly along three directions: refining experimental design[2-4], constructing suitable approximate model[5-7] and better sub-region management approach[2,4,6,8-10] to overcome these obstacles and to develop a sequential response surface modeling[4,6,8-10] to improve iteratively the approximate accuracy and optimization efficiency.

This paper presents an efficient sequential approximate modeling called Sequential quadratic Response Surface Method (SRSM) based on inherited Uniform Design(UD)[11]. The SRSM mainly consists of three distinctive aspects. Firstly, it constructs a low precision global response surface using UD in the design domain. Secondly, it determines the initial sub-region of sequential quadratic response surface through global response surface, and reuses previous sampling points for constructing the sequential response surface. Finally, it implements the subregion management based on the relative position of normalized optimum, so the approximate accuracy is iteratively improvement in optimization process and the global convergence is guaranteed.

\section{SeQuential ReSPONSE Surface Modeling}

\section{A. Design of Experiment}

UD as a multifactor Design of Experiment, is proposed by K.T. Fan[11] to seek sample points that are uniformly scattered on the design domain. UD firstly generates the samples according to Uniform Table(UT) notated as $\mathrm{U}_{p}\left(q^{n}\right)$, where $\mathrm{U}$ represents $\mathrm{UD}, p$ is the number of sample points, $q$ the number of levels for each factor, and $n$ the number of variables. A $p \times n$ matrix $\boldsymbol{U}=\left(u_{i, j}\right)$, where $u_{i, j}(i=1, \mathrm{~K}, p, j=1, \mathrm{~K}, n)$ is the $j$-th variable values of the $i$-th sample point in UT, is called U-type design. Then, $\boldsymbol{x}_{i}$, the actual values of the $i$-th sample point in the design domain is defined as

$$
\boldsymbol{x}_{i}=\boldsymbol{x}_{\mathrm{L}}+\left(\boldsymbol{U}_{i}-\boldsymbol{E}\right) \mathrm{o}\left(\boldsymbol{x}_{\mathrm{U}}-\boldsymbol{x}_{\mathrm{L}}\right) / n \quad i=1, \mathrm{~L}, p
$$

where $\boldsymbol{U}_{i}$ is the column vector values of the $i$-th sample point in $\mathrm{UT}, \boldsymbol{E}=(1, \mathrm{~L}, 1)^{\mathrm{T}}$ is the $n$ dimensional vector. 
„o $\mathrm{O}^{\text {ee }}$ is Hadamard multiplier. $\boldsymbol{x}_{\mathrm{U}}$ and $\boldsymbol{x}_{\mathrm{L}}$ are the upper and lower limit of $\boldsymbol{x}$, respectively.

\section{B. Approximate Method}

Polynomial response surface is one of the most popular approximate models utilized effectively in a variety of applications, because it can be easily constructed and quick convergence in optimization. A quadratic polynomial response surface model has the form

$$
y(\boldsymbol{x})=\beta_{0}+\sum_{i=1}^{n} \beta_{i} x_{i}+\sum_{1 \leq i \leq j \leq n}^{n} \beta_{i j} x_{i} x_{j}
$$

where $y(\boldsymbol{x})$, with $\boldsymbol{x}=\left(x_{1}, \mathrm{~K}, x_{n}\right)^{\mathrm{T}}$, is the predicted value. $x_{i} \quad(i=1, \mathrm{~K}, n)$ is the $i$-th design variable. $\beta_{0}, \beta_{i}$ and $\beta_{i j}(1 \leq i \leq j \leq n)$ are undetermined coefficients of quadratic polynomial, total $N_{\min }=(n+1)(n+2) / 2 \quad$. The coefficient $\boldsymbol{\beta}=\left(\beta_{0}, \beta_{1}, \mathrm{~L}, \beta_{p}\right)^{\mathrm{T}}$ is calculated with least squares regression, by minimizing the sum of squares of the errors between the predicted values $y(\boldsymbol{x})$ and the analysis values $f(\boldsymbol{x})$, using the equation $\boldsymbol{\beta}=\left(\boldsymbol{X}^{\mathrm{T}} \boldsymbol{X}\right)^{-1} \boldsymbol{X}^{\mathrm{T}} \boldsymbol{f}$. Where $\boldsymbol{X}$ is the matrix of variable data at the sample points, $\boldsymbol{f}=\left[f\left(\boldsymbol{x}_{1}\right), f\left(\boldsymbol{x}_{2}\right), \mathrm{L}, f\left(\boldsymbol{x}_{p}\right)\right]^{\mathrm{T}}$ the corresponding analysis values at each sample point. The equation $p \geq N_{\text {min }}$ is necessary to keep matrix $\boldsymbol{X}^{\mathrm{T}} \boldsymbol{X}$ nonsingularity.

\section{Model Management Approach}

Sizes of sequential sub-regions are highly influential on the approximate accuracy of model. When the approximate accuracy is not enough to ensure model convergence to a true optimum, the size of the sub-region should be zoomed and the position be adjusted. Model management approach proposed in the paper is composed of planning of the initial sub-region and moving and zooming of the sequential sub-region. The position of the initial sub-region is determined by the point with smallest value of the sample set, which contains the optimum $\boldsymbol{x}_{\mathrm{opt}}^{0}$ of global response surface. And the size is determined by the space around the point with smallest value and containing the $N_{\min }+1$ sample points, as shown in Fig .1. The "•"denotes the sample points designed by $\mathrm{U}_{13}\left(13^{2}\right)$, the "** "denotes $\boldsymbol{x}_{\mathrm{opt}}^{0}$ and the gray area including seven points is the initial sub-region. Space mapping of the sub-region to the normalized space $[-1,1]$ is used in optimization process to eliminate the variable scale effect on approximate accuracy and to unify the sequential response surface modeling.

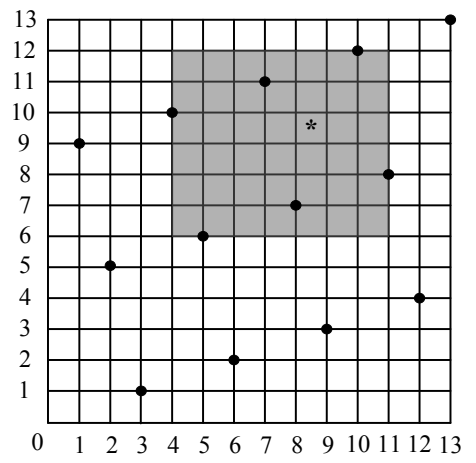

Figure 1. The initial sub-region

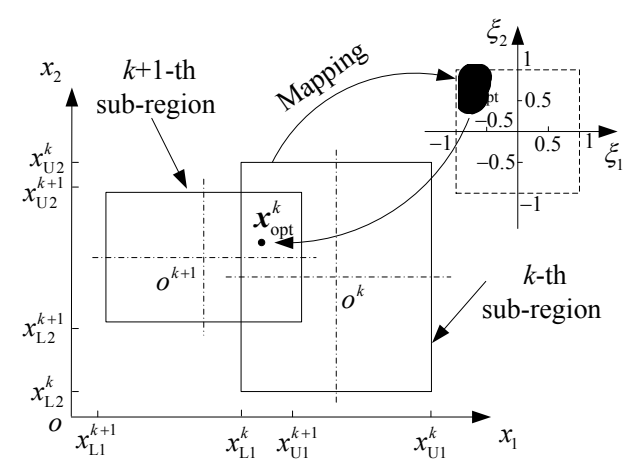

Figure 2. The moving and zooming of $\mathrm{k}+1$-th sub-region

Updating scheme of the moving and zooming of subregion is shown as Fig .2 for two variables problem. The size of the $k+1$-th sub-region is a proportion of the size of the $k$-th sub-region, and the proportional parameter $\alpha_{i}^{k+1}$ is calculated based on the normalized optimum $\xi_{i}^{k}$ as below

$$
\alpha_{i}^{k+1}=0.8+0.125 \tan \left[0.4 \pi\left(2\left|\xi_{i}{ }^{k}\right|-1\right)\right] \quad k=1,2, \ldots
$$

the values of $\alpha_{i}^{k+1}$ is limited within [0.42, 1.18]. $\alpha_{i}^{k+1}=0.8$ for shrinking slowly the size of the $k+1$-th subregion when $\xi_{i}^{k}$ nears to 0.5 or -0.5 . If $\xi_{i}^{k}$ is close to $1,-1$ or zero, the $\alpha_{i}^{k+1}$ should get 1.18 or 0.42 respectively to fast zoom the size of the $k+1$-th sub-region. The curve of parameter $\alpha_{i}^{k+1}$ versus the normalized optimum $\xi_{i}^{k}$ is shown as Fig .3. The $k+1$-th sub-region size $s_{i}^{k+1}$ is chosen from the smaller value between $a_{i}^{k+1} s_{i}^{k}$ and $s_{i}^{1}$, where $s_{i}^{k}$ and $s_{i}^{1}$ are the size of the range of the $i$-th variable in $k$-th sub-region and initial sub-region, respectively, and can be formula as

$$
s_{i}^{k+1}=\min \left\{a_{i}^{k+1} s_{i}^{k}, s_{i}^{1}\right\} \quad k=1,2, \mathrm{~K}
$$

The center position $o^{k+1}$ of the $k+1$-th sub-region is determined by the optimum $\boldsymbol{x}_{\mathrm{opt}}^{k}$ and the normalized optimum $\xi_{\text {opt }}^{k}$ of the $k$-th sub-region. Utilizing $s_{i}^{k+1}$, the upper and lower bounds of the $i$-th variable for $k+1$-th sub-region can be determined by 


$$
\begin{gathered}
\left\{\begin{array}{l}
x_{\mathrm{U} i}^{k+1}=\min \left(x_{i}^{* k}+0.5 \times\left[1+\psi_{i}^{k+1}\right] \times s_{i}^{k+1}, x_{\mathrm{U} i}\right) \\
x_{\mathrm{L} i}^{k+1}=\max \left(x_{i}^{* k}-0.5 \times\left[1-\psi_{i}^{k+1}\right] \times s_{i}^{k+1}, x_{\mathrm{L} i}\right)
\end{array}\right. \\
\psi_{i}^{k+1}=0.5 \times \max \left(0,\left|\xi_{i}^{k}\right|-0.5\right) \times \operatorname{sign}\left(\xi_{i}^{k}\right)
\end{gathered}
$$

where the $x_{i}^{* k}$ is the optimum of the $i$-th variable in the $k$ th sub-region. The $x_{\mathrm{U} i}$ and $x_{\mathrm{L} i}$ are the upper and lower bounds of the $i$-th variable for the design domain. The $\operatorname{sign}(\cdot)$ is sign function. The offset of the center of the $k+1$-th sub-region from the optimum $x_{i}^{* k}$ is controlled by $\psi_{i}^{k+1}$, which indicates the relative position of normalized optimum $\xi_{i}^{k}$ from the 0.5 or -0.5 , and the offset direction is decided by the symbol of $\xi_{i}^{k}$. The offset in (5) and (6) reduces the overlap of two adjacent sub-regions and accelerates the optimization convergence.

The convergence criteria of the optimization process is

$$
\left\{\begin{array}{l}
\left|f\left(\boldsymbol{x}_{\mathrm{opt}}^{k+1}\right)-f\left(\boldsymbol{x}_{\mathrm{opt}}^{k}\right)\right| / f\left(\boldsymbol{x}_{\mathrm{opt}}^{k}\right) \leq 1 \% \\
\left\|\boldsymbol{x}_{\mathrm{opt}}^{k+1}-\boldsymbol{x}_{\mathrm{opt}}^{k}\right\| /\left\|\boldsymbol{x}_{\mathrm{opt}}^{k}\right\| \leq 1 \%
\end{array}\right.
$$

where $f\left(\boldsymbol{x}_{\mathrm{opt}}^{k+1}\right)$ and $f\left(\boldsymbol{x}_{\mathrm{opt}}^{k}\right)$ are the optimum values for the $k+1$-th and $k$-th sub-regions, respectively.

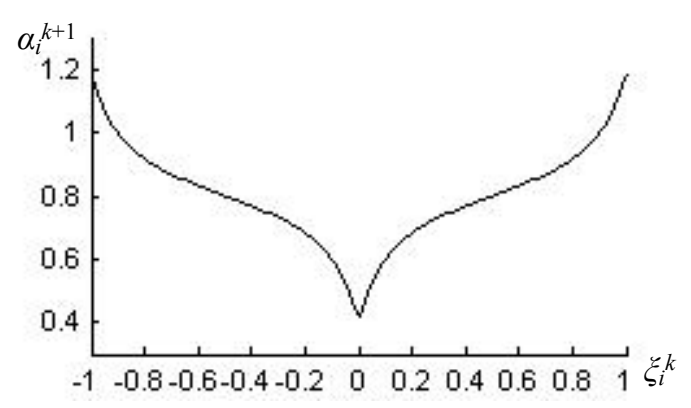

Figure 3. The curve of $\alpha_{i}^{k+1}$ vs. $\xi_{i}^{k}$

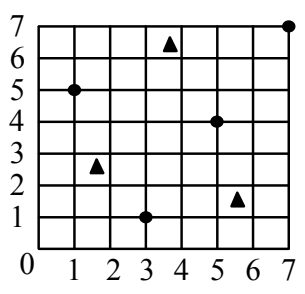

(a)

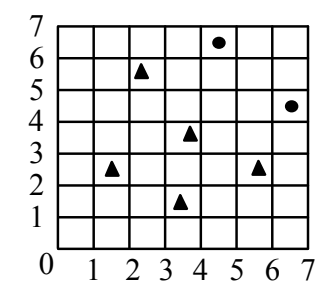

(b)
Figure 4. Inherited sample points in the sub-region: (a) $\mathrm{Ne}<0.6 \mathrm{Nmin}$; (b) $0.6 \mathrm{Nmin}<\mathrm{Ne}<\mathrm{Nmin}$

\section{Inherited Method}

The total sample size evaluated in the optimization process consists of the initial sample size in the design domain and the supplemental sample size generated in iterative process. It is necessary to elaborately choose the appropriate amount of initial samples for reducing the total sample size and improving the efficiency. In this work, the economy initial sample size is chosen as $1.5 N_{\min }$ based on literature[2-3] and experimental test.

In the optimization process. the sequential optimum is added to the sample set and the point in the set will be inherited as long as it falls into the sub-region. The sequential response surface model is fitted by no less than $N_{\text {min }}+1$ samples in the sub-region. If $N_{\mathrm{e}}$, which denotes the number of inherited points, is less than $N_{\min }+1$, the additional points will be generated as follow: When $N_{\mathrm{e}}$ is less than $0.6 N_{\min }$, create $N_{\min }$ sampling points by UD and remove the ones nearing the inherited points until remaining $N_{\min }+1$ points. When $\mathrm{N}_{\mathrm{e}}$ falls into $\left[0.6 N_{\min }\right.$ $\left.N_{\text {min }}\right]$, design the additional points by the principle of maximizing the minimum distance. For two variables, the sampling points were generated as shown in Fig 4 . The " $\Delta$ " denotes inherited points and the "•" denotes additional points.

\section{E. Optimization Procedure of SRSM}

Combined the NSGA-II algorithm[12] as RSM suboptimizer to obtain global optimum, The overall system optimization procedure of the proposed SRSM is represented as following:

Step 1. Define the design variables, the objectives and the constraints of optimization problem.

Step 2. Set $k=0$, Generate $1.5 N_{\min }$ sample points by $\mathrm{UD}$ in design domain, evaluate the function values at these points and record these points into the sample set.

Step 3. Construct the quadratic polynomial model by least square regression and execute an approximate optimization by NSGA-II to get an optimum $\boldsymbol{x}_{\mathrm{opt}}^{k}$.

Step 4. Evaluate actual function value at $\boldsymbol{x}_{\mathrm{opt}}^{k}$ and add $\boldsymbol{x}_{\mathrm{opt}}^{k}$ to the sample set. if $k=0$ then select the reference point with the smallest value in the sample set, define the initial sub-region that contains $N_{\min }+1$ points around the reference point with the minimum distance and go to Step 6.

Step 5. If the process satisfies the convergence criteria (7), stop the program. Otherwise, calculate the $k+1$ th sub-region by (3)-(6).

Step 6. Generate $N_{\min }+1$ sample points based on the inherited points $N_{\mathrm{e}}$ at the $k+1$-th sub-region: If $N_{\mathrm{e}}<0.6 N_{\text {min }}$, create $N_{\text {min }}$ candidate points by UD and maintain $N_{\min }+1$ sample points through removing the candidate ones close to the inherited points, as shown in Fig .5(a).

If $0.6 N_{\min } \leq N_{\mathrm{e}}<N_{\min }$, generate the supplementary points by the principle of maximizing the minimum distance, as shown in Fig .5(b).

If $N_{\mathrm{e}} \geq N_{\min }$, no sample points would be added.

Step 7. Set $k=k+1$ and go to Step 3 .

\section{NUMERICAL EXAMPLES}

The proposed SRSM has been tested with two widely accepted test functions and a engineering design. First of all, the test functions is performed to verify an accuracy and efficiency of the SRSM. 


\section{A. Test functions}

(1) Six-hump Camelback function(SC)

$$
\begin{gathered}
f_{\mathrm{SC}}=4 x_{1}^{2}-2.1 x_{1}^{4}+\frac{x_{1}^{6}}{3}+x_{1} x_{2}-4 x_{2}^{2}+4 x_{2}^{4} \\
x_{1,2} \in[-2,2]
\end{gathered}
$$

(2) Rastrigin function(RS)

$$
\begin{gathered}
f_{\mathrm{RS}}=x_{1}^{2}+x_{2}^{2}-\cos 18 x_{1}^{2}-\cos 18 x_{2}^{2} \\
x_{1,2} \in[-1,1]
\end{gathered}
$$

(3) Nonlinear function(NL)

$$
\begin{aligned}
f_{\mathrm{NL}}= & {\left[0.8 \sqrt{x_{1}^{2}+x_{2}^{2}}+0.35 \sin \left(\frac{2.4 \pi \sqrt{x_{1}^{2}+x_{2}^{2}}}{\sqrt{2}}\right)\right] } \\
& \left\{1.5 \sin \left[1.3 \arctan \left(\frac{x_{1}}{x_{2}}\right)\right]\right\} \\
& x_{1} \in[0.01,1], x_{2} \in[0,1]
\end{aligned}
$$

The optimal results are tabulated in Table $1 . N_{\mathrm{f}}$ notates the number of function evaluations. From Table 1, it shows that first the SRSM can converge to a very nearglobal optimum. Second, the SRSM has achieved better accuracy with much fewer function evaluations than the ARSM-II[4]. For function NL, the SRSM needs fewer function evaluations at a same accuracy as the Radial Basis Functions (RBF)[5]. Therefore, the SRSM is efficient and high accuracy in terms of the number of computation-intensive function evaluations and the optimization results, respectively.

TABLE 1. Optimal results of three test problems

\begin{tabular}{cccccc}
\hline $\begin{array}{c}\text { Test } \\
\text { Func. }\end{array}$ & $\begin{array}{c}\text { \# Local } \\
\text { Min. }\end{array}$ & Anal. solu. & Method & Optimum & $\boldsymbol{N}_{\boldsymbol{f}}$ \\
\hline \multirow{2}{*}{ SC } & \multirow{2}{*}{6} & \multirow{2}{*}{-1.032} & SRSM & -1.032 & 41 \\
& & & ARSM II & -1.029 & 44 \\
RS & \multirow{2}{*}{50} & \multirow{2}{*}{2.000} & SRSM & -1.879 & 51 \\
& & & ARSM II & -1.854 & 60 \\
NL & \multirow{2}{*}{1.6519} & SRSM & 1.6519 & 29 \\
& & & RBF & 1.6519 & 38 \\
\hline
\end{tabular}

\section{B. Engineering design}

The following section will show how the SRSM is applied to buckling optimization of conical shell with hole as shown in Fig .5. Shell wall thickness is $0.8 \mathrm{~mm}$. Material modulus of elasticity is $204 \mathrm{GPa}$. and P denotes the pressure load on the structure. The hole boundary is described as the super-elliptic function in parametric form as[13]

$$
\left\{\begin{array}{l}
x=x_{0}+a \cos ^{2 / \eta}(\theta) \\
y=y_{0}+b\left(1+c a \cos ^{2 / \eta}(\theta)\right) \sin ^{2 / \eta}(\theta)
\end{array}(0 \leq \theta \leq 2 \pi)\right.
$$

where the shape parameter $\eta$, distortion factor $c$ and the ellipse axes $a$ or $b$ constitute the design variables. Based on the parametrical mapping method[14], the parameterized geometry model with hole is established and the structural stability analysis is conducted using the finite element method. Maximization of conical shell carrying capacity can be expressed as

$$
\begin{aligned}
\operatorname{Max} & \mathrm{P}_{\mathrm{cr}}(\boldsymbol{x}) \quad \boldsymbol{x}=[a, \eta, c]^{\mathrm{T}} \in R^{3} \\
\text { s.t. } & A(\boldsymbol{x})=80384 \\
& 60 \leq a \leq 300 \\
& 2 \leq \eta \leq 6 \\
& -0.0033 \leq c \leq 0.0033
\end{aligned}
$$

where the objective $\mathrm{P}_{\mathrm{cr}}(\boldsymbol{x})$ is the critical buckling load of conical shell. The constraint $A(\boldsymbol{x})$ is for maintaining constant area of hole in conical shell.

Table 2 shows the optimization results for SRSM and NSGA-II. The optimal hole shape is shown in Fig .6. Population size and genetic algebra is set to 40 and 30 respectively in NSGA-II. The optimal results show that the SRSM only requires 5 iterations with 71 function evaluations to reach the optimum, whereas the NSGA-II algorithm needs 1200 function evaluations. The SRSM reduced function evaluations dramatically at a loss of little accuracy compared with the NSGA-II.

TABLE 2. Comparison of optimal results for SRSM and NSGA-II

\begin{tabular}{cccccc}
\hline Methods & $\begin{array}{c}\text { Optimal design } \\
(\boldsymbol{a}, \boldsymbol{\eta}, \boldsymbol{c})\end{array}$ & $\begin{array}{c}\text { Predicted } \\
\text { values( } \boldsymbol{N})\end{array}$ & $\begin{array}{c}\text { Analysis } \\
\text { values(N) }\end{array}$ & errors & $\boldsymbol{N}_{\boldsymbol{f}}$ \\
\hline SRSM & $(223.2,2.60,3.28)$ & 31123 & 29814 & $4.39 \%$ & 71 \\
NSGAII & $(247.8,2.48,3.27)$ & -- & 30764 & -- & 1200 \\
\hline
\end{tabular}

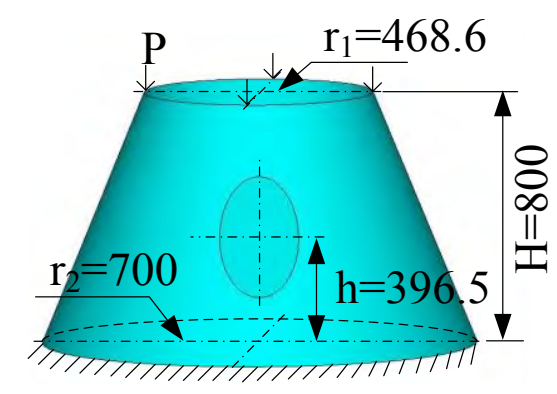

Figure. 5 Schematic of the conical shell with hole

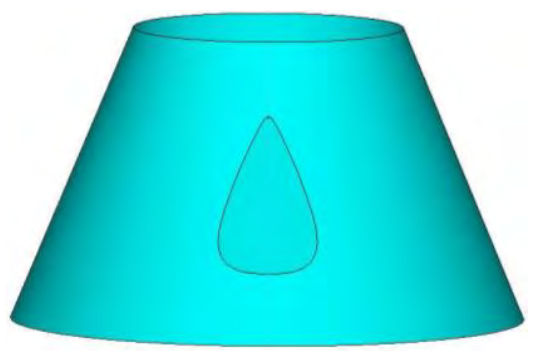

Figure. 6 The optimal hole shape on conical shell

\section{CONCLUSIONS}

In this paper, an efficient SRSM was presented using inherited UD as a design of experiment, a quadratic polynomial model as an approximate model, and a new move limit criteria as a model management approach. The 
SRSM achieves the high efficiency and accuracy because it reuses a previous data by using inherited UD and adjusts adaptively the sub-region to accelerate the convergence. Two functions and a design problem are solved and the availability of SRSM is proved by comparing the accuracy and efficiency of it with that of existing algorithms.

\section{ACKNOWLEDGMENT}

The authors gratefully acknowledge the support of the Natural Science Foundation of China (11362017), the Education Department Science and Technology Project of Jiangxi Province (GJJ13520, GJJ13494).

\section{REFERENCES}

[1] G. G. Wang, S. Shan, "Review of metamodeling techniques in support of engineering design optimization," J. Mech. Design,vol.129(2), pp.370-380, 2007.

[2] N. Stander, K. J. Craig, "On the robustness of a simple domain reduction scheme for simulation-based optimization," Eng. Comput., vol.19(4), pp.431-450,2002.

[3] T. W. Simpson, D. K. J. Lin, W. Chen, "Sampling Strategies for Computer Experiments: Design and Analysis," Int. J. Reliab. Appl., vol2(3), pp. 209-240, 2003.

[4] G. G. Wang, "Adaptive response surface method using inherited latin hypercube design points," J. Mech. Design, vol. 125,pp. 210220, 2003.

[5] T. Krishnamurthy, "Response Surface Approximation with augmented and compactly supported radial basis functions," 44th
AIAA/ASME/ASCE/AHS Structures, Structural Dynamics, and Materials Conference, Norfolk, USA, April 2003, AIAA-20031748 .

[6] D. Y. Li, Y. H. Peng, J. L. Yin, "Optimization of metal-forming process via a hybrid intelligent optimization technique," Struct Multidisc Optim, vol.34, pp.229-241,2007.

[7] J. Zhang, S. Chowdhury, A. Messac, "An adaptive hybrid surrogate model," Struct. Multidisc. Optim., vol.46, pp. 223-238,2012.

[8] V. M. P'erez, J. E. Renaud, L. T. Watson, "An interior-point sequential approximate optimization methodology," Struct. Multidisc. Optim., vol. 27, pp.360-370, 2004.

[9] S. Pajunen, O. Heinonen, "Automatic design of marine structures by using successive response surface method," Struct Multidisc Optim,vol.49, pp.863-871, 2014.

[10] Lee Yongbin, Jung Sunghun, Choi Dong-Hoon, "Progressive quadratic response surface modeling using inherited latinhypercube design," 11th AIAA/ISSMO Multidisciplinary Analysis and Optimization Conference, Portsmouth, USA, September, 2006, AIAA-2006-7092

[11] K. T. Fang, "Experimental Design by Uniform Distribution," Acta Math. Appl. Sinica, vol.3, pp.363-372,1980.

[12] K. Deb, A. Pratap, S. Agarwal, et al., "A fast and elitist multiobjective genetic algorithm: NSGA-II," IEEE Trans. evol. comput., vol.6(2), pp.182-197,2002.

[13] N. L. Pedersen, "Optimization of holes in plates for control of eigenfrequencies," Struct. Multidisc. Optim., vol28. pp.1-10,2004.

[14] W. H. Zhang, D. Wang, J. G. Yang, "A parametric mapping method for curve shape optimization on 3D panel structures," Int. J. Numer. Meth. Engng., vol.84(4), pp.485-504,2010. 